\title{
Promise of Cyclin-Dependent Kinases 4/6 as Therapeutic Targets in Breast Cancer
}

\author{
Le Zhang ${ }^{1,2}$ and Chuanwei Yang ${ }^{3,4,{ }^{*}}$ \\ ${ }^{1}$ College of Computer and Information Science, Southwest University, Chongqing, China \\ ${ }^{2}$ Department of Biostatistics and Computational Biology, Center for Biodefense Immune Modeling, University of Rochester, USA. \\ ${ }^{3}$ Systems Biology, the University of Texas MD Anderson Cancer Center, Houston, USA \\ ${ }^{4}$ Breast Medical Oncology, the University of Texas MD Anderson Cancer Center, Houston, USA
}

*Corresponding author: Chuanwei Yang, Systems Biology, The University of Texas MD Anderson Cancer Center, Houston, TX, 77030, USA, Tel: 713-7945700; Email: yangpaul2011@yahoo.com

Received date: Jul 08, 2014, Accepted date: Sep 24, 2014, Published date: Sep 27, 2014

Copyright: (c) 2014 Zhang L, et al. This is an open-access article distributed under the terms of the Creative Commons Attribution License, which permits unrestricted use, distribution, and reproduction in any medium, provided the original author and source are credited.

\section{Introduction}

In 2014, an estimated 235,000 new patients will be diagnosed with breast cancer in the US alone and about 40,000 patients will die from this disease, making breast cancer one of the leading causes of cancer death among women [1]. Accordingly, identifying new molecular targets which are druggable is of critical importance in breast cancer research and treatment. Breast cancer is commonly classified into Her2 positive, ER/PR positive and triple negative subtypes in clinic. While inhibitors of cyclin-dependent kinases $4 / 6$ are promising for the treatment of all breast cancers where the targeted pathway is deregulated, we focus on the Her2 positive breast cancer in this article.

Her2, also called erbB2, Neu, is a powerful oncogene in breast carcinogenesis [2]. Her2 positive subtype accounts for $20-30 \%$ of all breast cancer worldwide [3]. Although both antibody-based and small molecule-based medicine such as Herceptin and Lapatinib respectively have been developed in the past decade during the course of finding cures against Her2 positive breast cancer, there are limitations in the use of these medicine in clinic. Some patients are not responsive to the therapy and some other patients develop resistance to the therapy quickly [4,5]. Therefore new therapies on new targets are needed either as a single agent or in combination with currently existent therapies to combat Her2 positive breast cancer. Studies over the years have provided ample evidence indicating that CDK4/6 could be our important new therapeutic target in the fight against Her2-positive breast cancer [6]. CDK4/6 kinases are connected to Her2 signaling pathway through their interacting partner cyclin D1 (Figure 1). P16ink4a can bind cyclin D1 and CDK4/6 complexes and inhibit their kinase activities. Resistance to CDK4/6 inhibition can occur through $\mathrm{Rb}$ deficiency or $\mathrm{Rb}$ loss, reduced expression of CDK2 inhibitors p 1 and p27 and through events which augment the protein level or function of the individual component such as cyclin D1 and CDK4/6 in the pathway. Together cyclin D1-CDK4/6-p16 axis mediates Her2 signaling events in normal development and in mammary tumorigenesis [7] (Figure 1).

\section{Essential Role of Cyclin D1-CDK4/6-P16ink4a Axis in Her2 Positive Breast Cancer}

Studies using transgenic mouse model established the essential role of cyclin D1 and cdk4/6 in Her2 induced mammary tumor. In one study, it was demonstrated that ErbB-2 or ras oncogenes failed to induce mammary tumors in cyclin D1 null mice although cyclin D1deficiency did not hinder tumorigenesis by other oncogenes such as c- myc or Wnt-1 in mice in the same study [8]. Cyclin D1 interacts with many protein partners including CDK4, CDK6 and estrogen receptor etc. $[9,10]$. It can function through both kinase-dependent and kinaseindependent pathways [9]. Since knock-in of cyclin D1-KE mutant which renders kinase deficient in the cyclin D1KE/KE/ CDK4/6 complex was unable to restore the Her2 induced tumorigenesis in the wild type cyclin D1 deficient background in mice, requirement of CDK4/6 kinase activity in Her2 mammary tumorigenesis is indicated [7]. Among its interacting partners, Cyclin D1 can bind and activate both CDK4 and CDK6 [11].

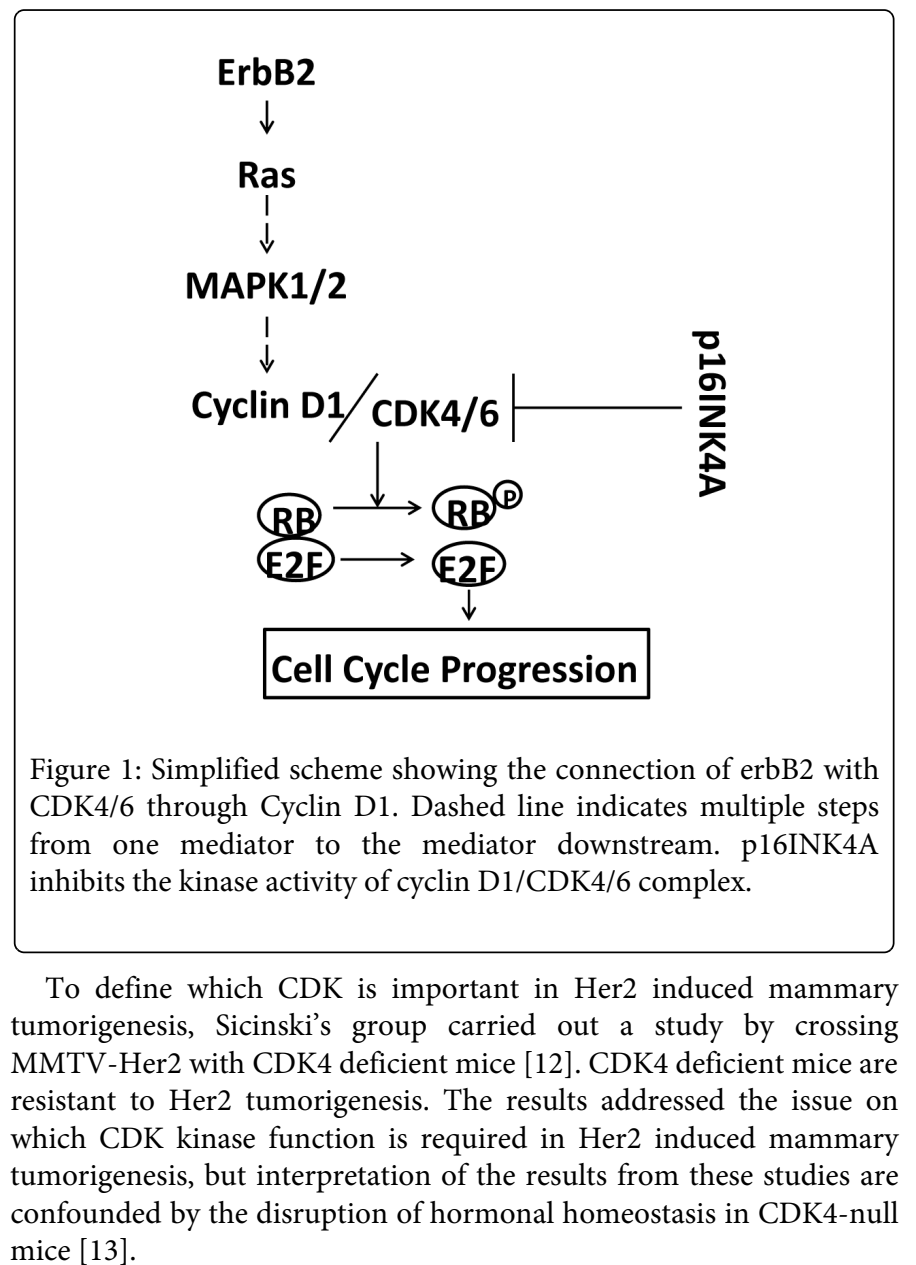


To overcome this problem, we created MMTV-p16ink4a transgenic mice which express p16ink4a, a natural inhibitor of CDK4/6 kinases, in mouse mammary gland. We crossed MMTV-p16ink4a with MMTV-ErbB2 transgenic mice to create bigenic MMTV-ErbB2-p16 mice under the leadership of Dr. Emmett Schmidt [11]. These bigenic mice do not display any of the developmental defects seen in CDK4null mice [13], but expression of p16ink4a blocked erbB2-induced mammary tumors in mice. It should be pointed out that some tumors did occur in erbB2-overexpressing mouse mammary gland under the condition of either cyclin D1-null, or CDK4-null or p16 expressing after a long latency [11]. The occurrence of tumors after a long delay is considered a sign of resistance to the inhibitors of cyclins or CDKs. Taken together, these experiments establish the role of CDK4/6 as critical mediator of erbB2 initiated tumorigenesis.
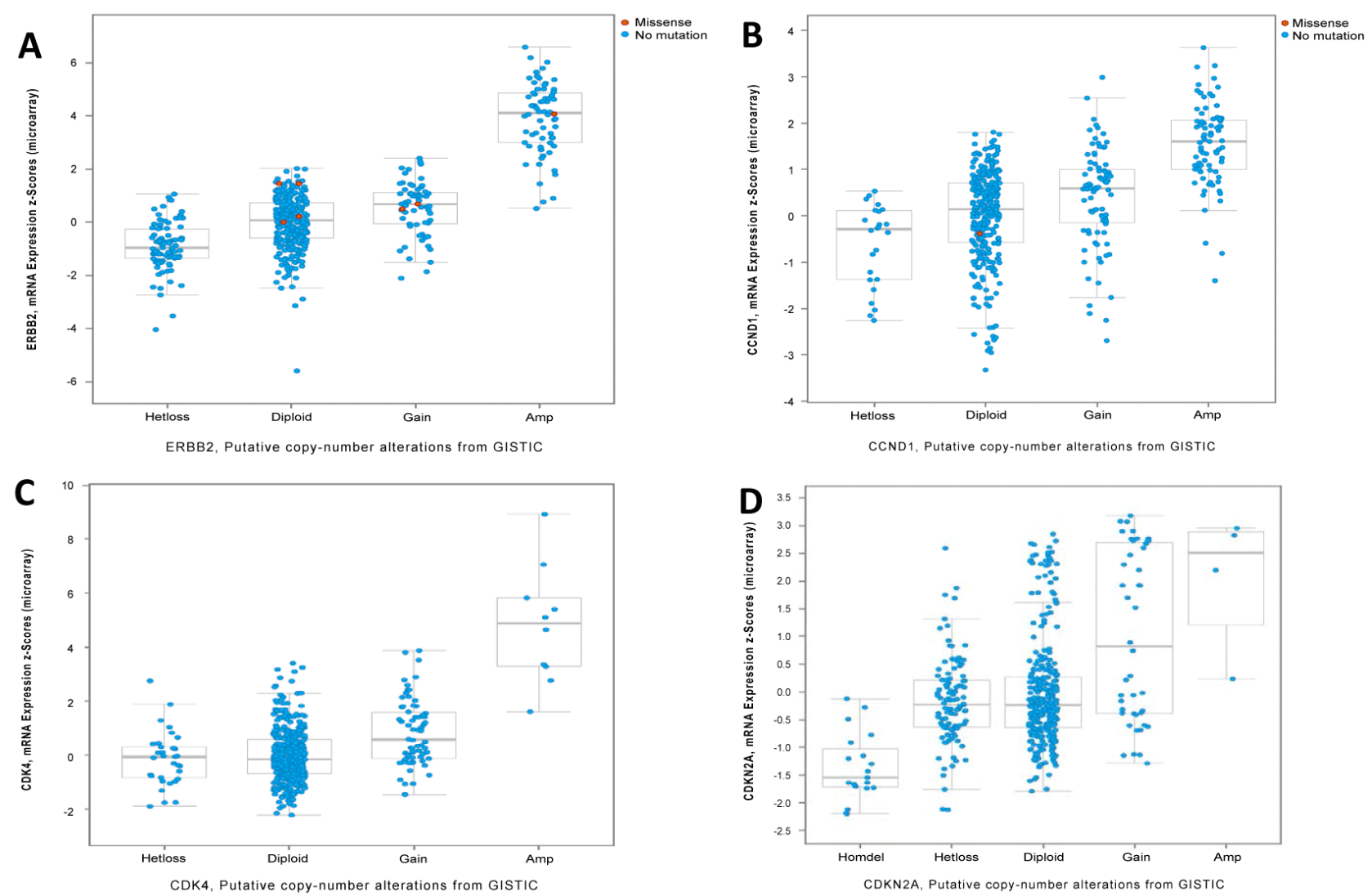

Figure 2: Correlation of gene copy number change with mRNA expression. Amp: gene amplification. Homdel: homozygous deletion. Hetloss: heterozygous loss. Each dot represents values from onepatient case. A. ErbB2 B. CCND1 (cyclin D1) C.CDK4. D.CDKN2A (p16).

\section{Deregulated Expression of Cyclin D1-CDK4/6- P16INK4a Axis is a Common Event in Breast Cancer.}

Deregulated expression of cyclin D1, CDK4/6, p16ink4a in human breast cancer has been reported by different research groups independently over the years $[11,14]$. With the easy public access to more and more clinically relevant data sets and the rapid development of data-mining tools, individual analysis of various components in a specific molecular pathway can be done conveniently on a data set. Using cBio Cancer Genomics Portal (http://cbioportal.org) created by scientists at Memorial Sloan-Kettering Cancer Center[15,16], we examined a data set containing 825 patient cases who were diagnosed with breast invasive carcinoma (TCGA, Nature 2012), of which 482 cases had sequencing and copy number alteration (CNA) data available. Among the cyclin D1/CDK4/6/p16ink4a axis, CCND1 gene is frequently amplified in breast cancer, followed by $\mathrm{cdk} 4$ gene (Figures 2B and 2C). Out of 482 cases analyzed, 79 cases or $16.4 \%$ had CCND1 gene amplification and 10 cases or $2.1 \%$ had CDK4 gene amplification. On the other hand, the CDK4/6 inhibitor gene p16ink4a suffered frequent loss either as a result of homozygous deletion or heterozygous loss of one allele (Figure 2D). As predicted, erbB2, CCND1, CDK4/6 gene amplification is positively correlated with the increased mRNA expression (Figures 2A, 2B and 2C) whereas p16ink4a gene loss has markedly decreased mRNA expression (Figure 2D). Co-amplification of erbB2 and CCND1 are not uncommon. In this database analyzed, out of 482 cases, 12 cases have co-amplification of both erbB2 and CCND1 gene. Representative cases are plotted in figures $3 \mathrm{~A}$ and $3 \mathrm{~B}$. mRNA values for case \#TCGA-AQ-A04H are $E R B B 2=3.85$ and $C C N D 1=2.087$. mRNA values for case \#TCGA-A1$\mathrm{A} 0 \mathrm{SM}$ are erbB2 $=5.516$ and $\mathrm{CCND} 1=2.667$. On the other hand, 16 loss is relatively frequent. Out of 482 cases, 19 cases have p16 homozygous deletion and 3 cases have both erbB2 amplification and p16 homozygous deletion. Representative cases are plotted in figure $3 \mathrm{C}$ and 3D. mRNA values for case \#TCGA-AR-A0TQ are erbB2 $=4.94$ and CDKN2A (p16) $=-2.475$. mRNA values for case \#TCGA-E2-A14P are erbB2 $=5.354$ and $\mathrm{CDKN} 2 \mathrm{~A}(\mathrm{p} 16)=-3.15$. Overall, 132 cases among 
Page 3 of 4

482 analyzed or $27.4 \%$ had deregulation of the cyclin D1/CDK4/6/ p16ink4a axis either in the form of single gene alteration in the axis or multiple gene alteration in combination.

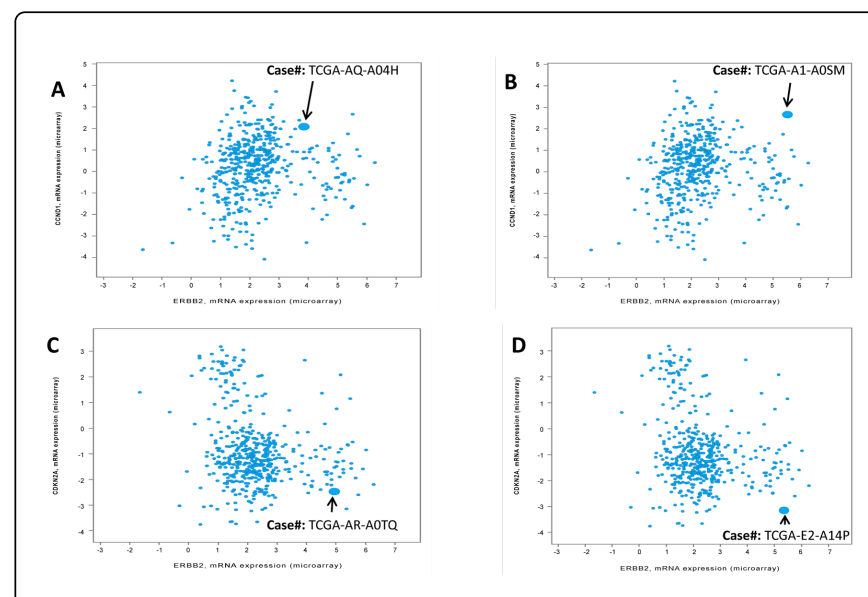

Figure 3: Cyclin D1 (CCND1) (A and B) or p16INK4A (CDKN2A) $(\mathrm{C}$ and $\mathrm{D})$ expression (Y-axis) Plotted against erbB2 mRNA expression (X-axis) for all tumors (cases). Each arrow points to a Representative case where there is either co-amplification of both erbB2 and cyclin D1 gene (A and B) Or ERBB2 gene amplification accompanied by p16 homozygous deletion $(\mathrm{C}$ and $\mathrm{D})$.

\section{Cyclin D1-Cdk4/6-P16ink4a Axis as Therapeutic Targets in Breast Cancer Treatment}

Over the last 20 years, attempts have been made to develop CDK inhibitors for clinical use. Flavopiridol (Alvocidib) was the first CDK inhibitor to enter clinical trials [17]. Reports from several phase II studies damped the enthusiasm of some investigators $[18,19]$. Another CDK inhibitor AT7519 developed by Astex Therapeutics Ltd [20] did not meet the expectations of the research community as well. This first generation of CDK inhibitors are considered broad in spectrum of CDK targets, not selective for specific CDK(s). Lessons learned from earlier experiments with CDK inhibitors have led to the development of newer generations of CDK inhibitors targeting CDK4/6 activities in breast cancer [9].

Palbociclib (PD0332991). Developed by Pfizer, Palbociclib inhibits cell proliferation in some breast cancer cell lines tested such as MCF7, T47D, ZR75-1 and some other non-breast cancer cell lines such as Colo-205 (Colon), H1299(Lung) etc [21]. Finn RS et al. later broadened the cell line spectrum to include some Her2 positive breast cancer lines. Palbociclib inhibited cell growth of Her2 positive breast cancer cell lines such as BT474, SKBR3, MDA-MB-361 etc. with concentrations in the nanomolar range [22]. The potent selective inhibitory activity of Palbociclib on CDK4/6 kinases is demonstrated in vivo in an erbB2 overexpressing transgenic mouse model in which Its inhibitory effects on erbB2-induced murine mammary tumors phenocopied the effect of conditional cyclin D1 knockout, namely it inhibited tumor progression and induced senescence of cancer cells [23]. A phase II study combining Palbociclib and letrozole in the treatment of ER+/Her2- breast cancer yielded impressive results (2014 AACR abstract \#CT101). A phase III trial using the same combination for ER+/Her2- breast cancer is underway. If the drug is eventually approved by FDA for the treatment of breast cancer, clinical trials using Palbociclib as a single agent or in combination with other approved drugs such as Herceptin for the treatment of Her2 positive breast cancer could be predicted in the near future.

There are some other CDK4/6 inhibitors in development such as LEE011, Developed by Novartis, and LY2835219, Developed by Eli Lilly [9]. Both compounds are orally bioavailable and both have the potential to be used in breast cancer treatment [24,25]. Phase $1 \mathrm{~b} / 2$ trials of LEE011 (NCT 01857193) and LY2835219 (NCT02057133, NCT02102490) are currently recruiting patients (http:// clinicaltrials.org). Which CDK4/6 inhibitor will eventually enter the market depending on the results of later clinical trials. The good news is that we are making progress in the field.

\section{Conflict of Interest}

The authors declare no potential conflict of interest.

\section{Acknowledgements}

L. Zhang received support from the Natural Science Foundation of China under Grant No. 61372138, the Chinese Recruitment Program of Global Youth Experts, and USA NIH grant U01 CA166886-01.

\section{References}

1. Cancer facts and figures 2014

2. Hung MC, Schechter AL, Chevray PY, Stern DF, Weinberg RA (1986) Molecular cloning of the neu gene: absence of gross structural alteration in oncogenic alleles. Proc Natl Acad Sci U S A 83: 261-264.

3. Mitri Z, Constantine T, O'Regan R (2012) The HER2 Receptor in Breast Cancer: Pathophysiology, Clinical Use, and New Advances in Therapy. Chemother Res Pract 2012: 743193.

4. Giordano SH, Temin S, Kirshner JJ, Chandarlapaty S, Crews JR, et al. (2014) Systemic therapy for patients with advanced human epidermal growth factor receptor 2-positive breast cancer: american society of clinical oncology clinical practice guideline. J Clin Oncol 32: 2078-2099.

5. Gampenrieder SP, Rinnerthaler G, Greil R (2013) Neoadjuvant chemotherapy and targeted therapy in breast cancer: past, present, and future. J Oncol 2013: 732047.

6. Lee YM, Sicinski P (2006) Targeting cyclins and cyclin-dependent kinases in cancer: lessons from mice, hopes for therapeutic applications in human. Cell Cycle 5: 2110-2114.

7. Landis MW, Pawlyk BS, Li T, Sicinski P, Hinds PW (2006) Cyclin D1dependent kinase activity in murine development and mammary tumorigenesis. Cancer Cell 9: 13-22.

8. Yu Q, Geng Y, Sicinski P (2001) Specific protection against breast cancers by cyclin D1 ablation. Nature 411: 1017-1021.

9. Casimiro MC, Velasco-Velázquez M, Aguirre-Alvarado C, Pestell RG (2014) Overview of cyclins D1 function in cancer and the CDK inhibitor landscape: past and present. Expert Opin Investig Drugs 23: 295-304.

10. Yang C, Chen L, Li C, Lynch MC, Brisken C, et al. (2010) Cyclin D1 enhances the response to estrogen and progesterone by regulating progesterone receptor expression. Mol Cell Biol 30: 3111-3125.

11. Yang C, Ionescu-Tiba V, Burns K, Gadd M, Zukerberg L, et al. (2004) The role of the cyclin D1-dependent kinases in ErbB2-mediated breast cancer. Am J Pathol 164: 1031-1038.

12. Yu Q, Sicinska E, Geng Y, Ahnström M, Zagozdzon A, et al. (2006) Requirement for CDK4 kinase function in breast cancer. Cancer Cell 9: 23-32.

13. Rane SG, Dubus P, Mettus RV, Galbreath EJ, Boden G, et al. (1999) Loss of Cdk4 expression causes insulin-deficient diabetes and Cdk4 activation results in beta-islet cell hyperplasia. Nat Genet 22: 44-52. 
Citation: Zhang L and Yang C (2014) Promise of Cyclin-Dependent Kinases 4/6 as Therapeutic Targets in Breast Cancer. J Carcinog Mutagen

Page 4 of 4

14. Geradts J, Wilson PA (1996) High frequency of aberrant p16(INK4A) expression in human breast cancer. Am J Pathol 149: 15-20.

15. Cerami E, Gao J, Dogrusoz U, Gross BE, Sumer SO, et al. (2012) The cBio cancer genomics portal: an open platform for exploring multidimensional cancer genomics data. Cancer Discov 2: 401-404.

16. Choi YJ, Li X, Hydbring P, Sanda T, Stefano J, et al. (2012) The requirement for cyclin $\mathrm{D}$ function in tumor maintenance. Cancer Cell 22: 438-451.

17. Sedlacek H, Czech J, Naik R, Kaur G, Worland P, et al. (1996) Flavopiridol (L86 8275; NSC 649890), a new kinase inhibitor for tumor therapy. Int J Oncol 9: 1143-1168.

18. Shapiro GI, Supko JG, Patterson A, Lynch C, Lucca J, et al. (2001) A phase II trial of the cyclin-dependent kinase inhibitor flavopiridol in patients with previously untreated stage IV non-small cell lung cancer. Clin Cancer Res 7: 1590-1599.

19. Schwartz GK, Ilson D, Saltz L, O'Reilly E, Tong W, et al. (2001) Phase II study of the cyclin-dependent kinase inhibitor flavopiridol administered to patients with advanced gastric carcinoma. J Clin Oncol 19: 1985-1992.

20. Wyatt PG, Woodhead AJ, Berdini V, Boulstridge JA, Carr MG, et al (2008) Identification of N-(4-piperidinyl)-4-(2,6 dichlorobenzoylamino)-1H-pyrazole-3-carboxamide (AT7519), a novel cyclin dependent kinase inhibitor using fragment-based X-ray crystallography and structure based drug design. J Med Chem 51(16): 4986-4999.

21. Fry DW, Harvey PJ, Keller PR, Elliott WL, Meade M, et al. (2004) Specific inhibition of cyclin-dependent kinase 4/6 by PD 0332991 and associated antitumor activity in human tumor xenografts. Mol Cancer Ther 3: 1427-1438.

22. Finn RS, Dering J, Conklin D, Kalous O, Cohen DJ, et al. (2009) PD 0332991, a selective cyclin D kinase 4/6 inhibitor, preferentially inhibits proliferation of luminal estrogen receptor-positive human breast cancer cell lines in vitro. Breast Cancer Res 11(5): R77.

23. Gao J, Aksoy BA, Dogrusoz U, Dresdner G, Gross B, et al. (2013) Integrative analysis of complex cancer genomics and clinical profiles using the cBioPortal. Sci Signal 6: pl1.

24. Gelbert LM, Cai S, Lin X, Sanchez-Martinez C, Del Prado M et al. (2014) Preclinical characterization of the CDK4/6 inhibitor LY2835219: in-vivo cell cycle-dependent/independent anti-tumor activities alone/in combination with gemcitabine. Invest New Drugs: 825-837

25. Dean JL, Thangavel C, McClendon AK, Reed CA, Knudsen ES (2010) Therapeutic CDK4/6 inhibition in breast cancer: key mechanisms of response and failure. Oncogene 29: 4018-4032. 